\title{
Passive internet-based crane teleoperation with haptic aids
}

\author{
Alejandro Fernández Villaverde*, Cesáreo Raimúndez, and Antonio Barreiro
}

\begin{abstract}
Crane operation is a challenging task, due to the combined problem of obstacle avoidance and load swing suppression in underactuated conditions. This paper presents a human-machine interface that increases the operator's perception of a gantry crane's workspace. With this aim, a virtual environment resembling the workspace is connected with a haptic device. This allows the user to receive not only visual but also tactile feedback, thus increasing maneuvering safety. Additionally, this capability is integrated in a teleoperation setup, adopting a passivity-based control approach that guarantees overall stability. This includes also the design of controllers by means of the IDA-PBC method. Experimental results carried out with a laboratory crane show its feasibility for internet-based teleoperation and demonstrate the improvements on the system performance.
\end{abstract}

Keywords: cranes, haptics, passivity, teleoperation

\section{INTRODUCTION}

This paper presents an overall stable setup for safely operating mechanical equipment placed in a remote location. With this aim, a passivity-based approach is adopted, and a gantry crane is chosen as the illustrating example.

Gantry or overhead cranes are widely used devices, very common in construction, transportation (harbors) and industry [1]. They are underactuated systems, since they have-assuming a simple model-5 degrees of freedom ( 3 cartesian coordinates and 2 deviation angles of the payload) and only 3 control inputs (the forces exerted by the motors on the girder, trolley, and lifting cable). Therefore, avoiding payload oscillations is a difficult task, see [12] for a recent example. Common approaches for addressing this problem include open-loop techniques such as input shaping. Input shaping is a command filtering method: it tries to reduce vibrations by modifying the input command $[4,26]$. A series of impulses are convolved with the input command, and the result is then used as the reference command for the system. An entirely different approach is the closed-loop framework, which can be adopted if the required sensors

Manuscript received - - 2010; revised - -, 2011; accepted - -, 2011. Recommended by Editorial Board member ... under the direction of ... . This work was supported by the Spanish Department of Science and Technology under grant DPI200407670-C02-01. A.F. Villaverde thanks A.J. van der Schaft for helpful comments, and Miguel Díaz-Cacho for collaboration with the experimental setup.

A.F. Villaverde is with the Process Engineering Group, IIMCSIC, 36208, Vigo, Spain (e-mail: afvillaverde@iim.csic.es).

Cesáreo Raimúndez and Antonio Barreiro are with the Department of Systems Engineering \& Control, University of Vigo, 36310 Vigo, Spain (e-mail: \{cesareo, abarreiro\}@uvigo.es).

* Corresponding author. are available. In that case, it is possible to apply classic feedback control techniques such as ProportionalDerivative (PD) control [14].

The ability to suppress payload oscillations is especially critical when time delays are present, as is the case in teleoperation. Teleoperated systems enable human operators to perform tasks on a plant placed in a remote location, and follow a master-slave scheme where a slave manipulator reproduces the movements of a master manipulator that is commanded by an operator. If this operator feels some resistance to motion which has a physical meaning related to the nature of the task, the performance of the teleoperated system can be improved, due to the fact that force feedback increases the feeling of telepresence. This is called bilateral teleoperation [11], in which the operator does not only provide position/velocity commands, but is also part of the control loop, sharing the control responsibility with the controllers. Modern bilateral teleoperation stems from [3], where it was shown that, in presence of time delays, the communications channel can become unstable if master and slave exchange power variables (force and velocity). Consequently, the use of wave variables-which are linear combinations of power variables-was advocated [16], since their transmission renders a communications channel with constant time delays passive. Passivity, which amounts to the absence of energy generation, is a sufficient condition to guarantee stability of a system. Passivity-based control methods have been the dominant approach for handling bilateral teleoperation, as shown in a recent tutorial [17]. Passivity-Based Control (PBC) is a modeling and control methodology capable of providing great insight to the control engineer, due to the fact that it handles intuitive concepts such as energy and power. Particularly, the Interconnec- 
tion and Damping Assignment Passivity Based Control (IDA-PBC) method [18] allows to simultaneously shape the potential and kinematic energy of a system, as well as to modify its interconnection and dissipation properties. Here it is applied to a gantry crane for the first time. If internet is used as the communications channel, the User Datagram Protocol (UDP) should be chosen [15]. This entails having the information delivered in data packets with time-varying delays, that may arrive in disorder and eventually get lost on the way. This can lead again to a loss of passivity unless an additional arrangement is made, such as the one presented in [5] which was first applied to crane teleoperation in [7]. We have adopted this solution due to its robustness and to the fact that it fits nicely into the passivity framework.

In the present work, both tactile and visual feedback is provided in order to help the crane operator. It is the first time that these capabilities are integrated in a HumanMachine Interface (HMI) for crane teleoperation. A virtual environment is created, generating repulsion forces around the obstacles which are rendered to the operator by means of a haptic device. Haptic devices [22] are machines capable of providing the user with tactile information and the sense of touch. They can be used for augmenting the force perceived by the user, giving him cues which are based on environment models or sensors. This is very useful in teleoperation, since it increases the sense of telepresence. Interest in haptics has been increasing in the last fifteen years, especially in medicine, although the use of these techniques is still not common in the industry or transportation. However, recent examples of applications of haptic feedback for teleoperation $[2,24]$ show that it is advantageous for increasing operation precision and safety. It is thus a promising area that deserves more research.

To sum up the introduction to the methods, we remark three main advantages of the passivity approach: (i) intuitive and physical principle, based on energy and power concepts; (ii) multidomain: it allows to integrate mechanical, electrical, and other subsystems; and (iii) hierarchical and modular: feedback interconnections of passive systems give rise to passive systems. These advantages of passivity are specially important in teleoperation applications: a teleoperation system is a complex system, with mechanical/electrical subsystems, time-delays, and interactions with humans and environment. In particular, the environment is usually passive, and a human operator intends to behave passively, feeling in a haptic sense when he/she is introducing damping in the system. Passivity provides also a systematic mechanism to ensure stability, by scrutiny of power interchange between subsystems. If nonpassive controllers are implemented, guaranteeing stability usually requires Lyapunov-Krasovskii functionals or other involved techniques.

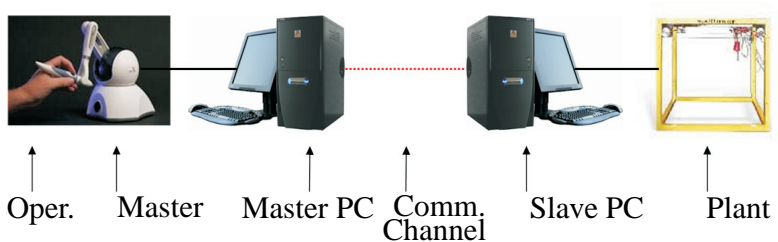

Fig 1: Hardware architecture

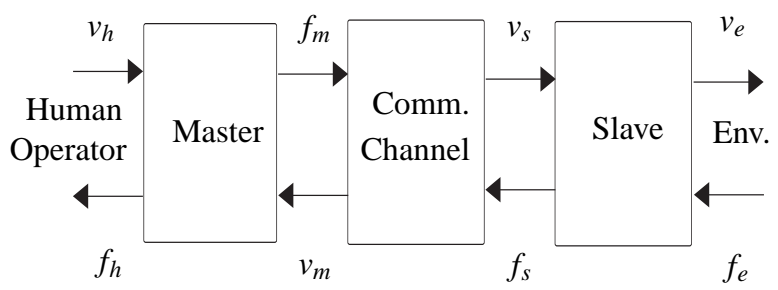

Fig 2: Teleoperation blocks

The structure of this paper is as follows: first, an overview of the system's logical, hardware and software architecture is given in section 2 . Then, the solutions adopted for ensuring a passive communications channel are described in section 3 . The master and slave controllers are obtained in section 4., and the HMI is presented in section 5. Experimental results are given in section 6., and the paper finishes with the conclusions and guidelines for future research.

\section{OVERALL SYSTEM ARCHITECTURE}

In a first approximation, the overall system of Fig. 1 can be described by the general block diagram of Fig. 2, which is valid for most bilateral teleoperation setups. It consists of three main subsystems: the master side (interacting with the human operator), the communication channel, and the slave side (interacting with the environment). The port variables are the force vectors $f_{i}$ and the velocities $v_{i}$, with $i \in\{h, m, s, e\}$, which are the indices for human, master, slave, and environment; their product amounts to the exchanged mechanical power. Their dimension is three, corresponding to the $(x, y, z)$ cartesian coordinates and to the number of actuated degrees of the gantry crane. The causality convention for inputs and outputs is given by Fig. 2 .

In a more detailed description, the system can be further decomposed into the blocks of Fig. 3 as follows. The slave side contains not only the plant dynamics (the crane), but also the crane controller. This controller can be used to modify the natural dynamics of the crane so that the perceived crane at the master side can be more easily manipulated. This adaptation might include different kinds of impedance balancing, damping injection, 


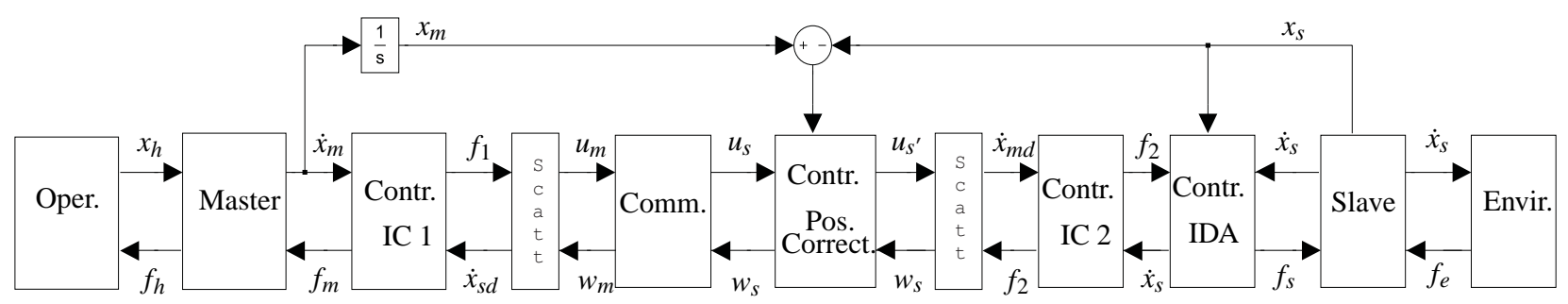

Fig 3: Logic structure

energy shaping, and so on. The design of this slave controller is described in section 4. The master side contains the human operator, the master device and the master controller, with a role similar to the role of the slave controller: adapting the dynamics to improve the masterslave interaction and manageability. Since in this case the master device is a light and frictionless haptic device, it is possible to neglect its dynamics. The communication channel contains the time-delay, which is generally unknown and time-varying; discrete time and information losses will also be taken into consideration. Details about how the communications are handled are given in the next section.

\subsection{Hardware}

The hardware components are shown on Fig. 1, where there are two elements that can be considered external: the human operator at the master side, and the work environment of the crane at the slave side (i.e., the crane workspace). The human operator handles a master manipulator, the Phantom Omni haptic device manufactured by SensAble Technologies. It is connected to a local computer, where the human-machine interface and the local controllers are implemented. The communications network splits the system in a local (master) and a remote (slave) side. Scattering blocks are placed at each side of the network; between them, wave variables are used, while power variables (force, velocity) are exchanged outside them (see next section for details). The slave device is the Inteco 3DCrane, which is connected to a second computer whose task is to read and send data through the communications channel, collect information from the plant sensors, implement the remote control loops and handle the actuators.

The crane can be seen in more detail on Fig. 4.A. It is a 3-dimensional, laboratory-scale gantry crane with 3 DC motors-one for each axis-as actuators and 5 sensors measuring the cart position on the horizontal plane $(x, y)$, the cable length $(\rho)$ and the 2 deviation angles of the payload $\left(\theta_{x}, \theta_{y}\right)$.

The SensAble Phantom Omni (Fig. 4.B) is an impedance device, that measures position of its tip and renders force to the user. It has 6 degrees of freedom, 3 of them for absolute positioning ( $x, y, z$ coordinates) and

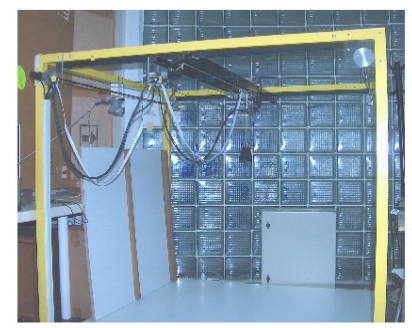

A

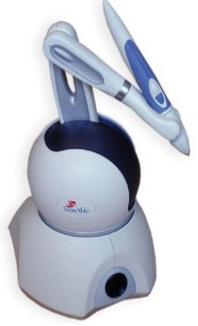

B
Fig 4: A) Inteco 3DCrane; B) Phantom Omni

3 additional for orientation. Besides, there are 2 buttons with which the user can specify additional actions.

\subsection{Software}

A Matlab/Simulink environment is used at both sides of the setup, both for development and implementation purposes. At the master side, the interface with the haptic device is carried out by means of the Handshake ProSense Virtual Touch toolbox, and the real-time requirements are met with Matlab's Real-Time Workshop toolbox. At the slave side, the computer-crane interface is done with Inteco's 3DCrane Control/Simulation Toolbox, which is integrated in a Simulink environment with the Humusoft Real-Time toolbox. This scheme, which is partially built upon the telehaptic architecture described in [20], is shown in Fig. 5.

\section{LINE PASSIVATION}

\subsection{The passivity approach}

The equations regarding power exchange of the system depicted in Fig. 2 are:

$$
\begin{aligned}
f_{s}^{\top}(t) v_{s}(t)-f_{e}^{\top}(t) v_{e}(t) & \geq \dot{H}_{s}(t) \\
f_{h}^{\top}(t) v_{h}(t)-f_{m}^{\top}(t) v_{m}(t) & \geq \dot{H}_{m}(t) \\
-f_{s}^{\top}(t) v_{s}(t)+f_{m}^{\top}(t) v_{m}(t) & \geq \dot{H}_{c}(t)
\end{aligned}
$$




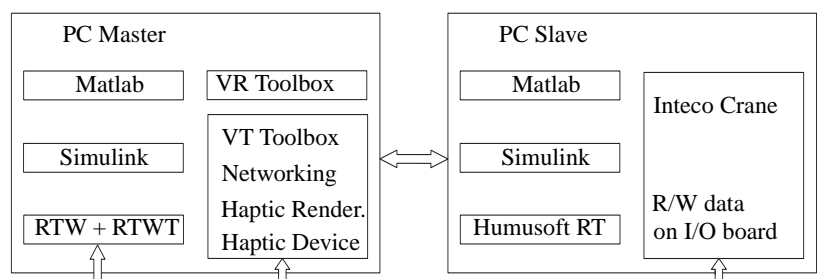

C Compiler Device API Sens. OpenHaptics Interface w. Omni

Omni Drivers

Fig 5: Software architecture

where $\dot{H}_{m}, \dot{H}_{s}$ and $\dot{H}_{c}$ are time derivatives, along the solutions of the system, of the Hamiltonians assigned for the master, slave and communication blocks. The master and slave blocks contain real and/or virtual finite dimensional mechanical dynamics. The equations impose passivity of these systems with respect to the storage functions $H_{m}, H_{s}, H_{c}$. By connecting the three blocks, it is straightforward to see that the global system is passive with respect to the total Hamiltonian $H_{m}+H_{s}+H_{c}$ :

$$
f_{h}^{\top}(t) v_{h}(t)-f_{e}^{\top}(t) v_{e}(t) \geq \dot{H}_{s}(t)+\dot{H}_{m}(t)+\dot{H}_{c}(t)
$$

Furthermore, if the operator and the environment are passive, then the complete system is bounded $\left(\dot{H}_{s}(t)+\right.$ $\left.\dot{H}_{m}(t)+\dot{H}_{c}(t) \leq 0\right)$. This is the basic idea of the approach: interconnecting passive systems so that the overall setup is itself passive and, therefore, stable.

\subsection{Scattering Transformation}

Since the classical results in [3] and [16] it is well understood that the non-passive character of the communication delays can be arranged using the scattering theory or the equivalent wave-variables approach.The underlying idea is mimicking the behavior of an analog lossless transmission line. In our application we adopt the scattering transformations (see Fig. 6):

$$
\left[\begin{array}{l}
u_{m} \\
v_{m}
\end{array}\right]=\left[\begin{array}{cc}
\sqrt{2} B^{-1 / 2} & -I \\
B^{-1} & -\sqrt{2} B^{-1 / 2}
\end{array}\right]\left[\begin{array}{c}
f_{m} \\
w_{m}
\end{array}\right]
$$

and

$$
\left[\begin{array}{c}
w_{s} \\
v_{s}
\end{array}\right]=\left[\begin{array}{cc}
\sqrt{2} B^{-1 / 2} & -I \\
-B^{-1} & \sqrt{2} B^{-1 / 2}
\end{array}\right]\left[\begin{array}{c}
f_{s} \\
u_{s}
\end{array}\right]
$$

where the matrix $B>0$ is the line impedance. The transmitted variables are no longer power variables (forces and velocities $\left.f_{i}, v_{i}\right)$, but wave variables $u_{i}, w_{i}$, so $u_{s}(t)=u_{m}(t-h), w_{m}(t)=w_{s}(t-h)$, where $h \geq 0$ is the communication delay. It can be seen that when $h=0$, the two scattering transformations cancel each other and forces and velocities are directly transmitted: $v_{s}(t)=v_{m}(t)$

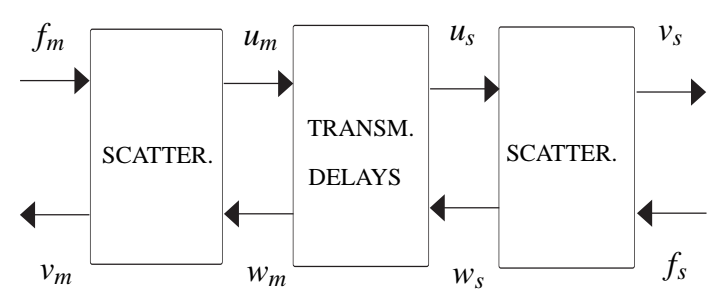

Fig 6: Line blocks

and $f_{m}(t)=f_{s}(t)$. When $h>0$ the transparency property $\left(v_{s}(t)=v_{m}(t), f_{m}(t)=f_{s}(t)\right)$ is degraded, but the great advantage is that the system is lossless with respect to the storage function

$$
H_{c}(t)=\frac{1}{2} \int_{t-h}^{t}\left(\left\|u_{m}(\tau)\right\|^{2}+\left\|w_{s}(\tau)\right\|^{2}\right) d \tau,
$$

this is, the integral of the power of the waves for the duration of the transmission. It easily follows that

$$
-f_{s}^{\top}(t) v_{s}(t)+f_{m}^{\top}(t) v_{m}(t)=\dot{H}_{c}(t)
$$

for every possible value of $h$.

These theoretical results are valid for continuous time and constant time delays. However, if some part of the teleoperation system is in discrete time, some additional considerations have to be made. In [23] it was shown how to discretise a port-Hamiltonian system preserving its passivity, and how to passively interconnect continuous and discrete time Hamiltonian systems. This is achieved by defining the flow through the interconnection port of the discrete system as $f_{d}(k):=$ $\frac{x(k T)-x((k+1) T)}{T}$, where $x(*)$ is the integral of the flow through the interconnection port of the continuous system. This trivial condition ensures that there is an exact matching between the energy going into the continuous time system and the one coming from the discrete time system. Additionally, it was shown that a scatterized communication channel with fixed transmission delays is not only lossless in continuous time but also in discrete time.

With these considerations, passivity of the communications channel is guaranteed for any constant time delay in continuous or discrete time. This means that no such delay can make the system unstable.

\subsection{Data Handling in Internet-based Teleoperation}

When a packet-switched network such as internet is chosen as the communication channel, the delay is not constant and passivity cannot be assumed. Furthermore, additional undesirable effects can appear depending on which protocol is selected. In [15] it was shown that the UDP protocol, although not confirmation based, is more suitable for control purposes than TCP/IP, for it 


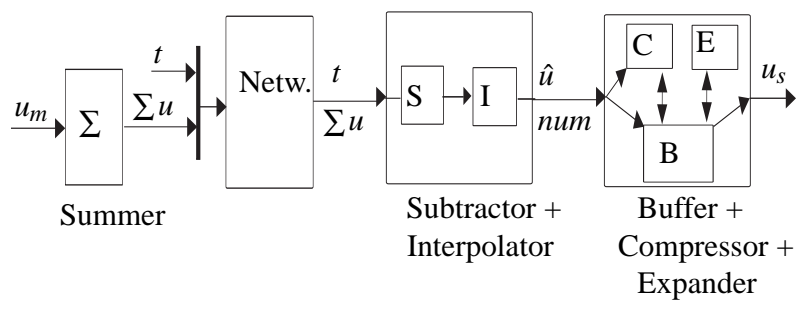

Fig 7: Data handling of the UDP communications

preserves the shape of the sent wave with more accuracy. Its main disadvantage, however, is that it fails to guarantee the order of arrival of the information packets, and some of them can be lost on the way. Thus, the issues of variable time delay, disordered packets and loss of information must be addressed; all of them can destabilize the system if not properly treated. Perhaps the more general arrangement for dealing with these issues is the one presented in [5], which manages to guarantee passivity and improve tracking performance. The basic idea of this arrangement is as follows.

Suppose that a data packet is lost, resulting in an empty sampling instance. By the scattering transformation, and from (3), the transmitted variables $u_{m}(t)$ and $w_{s}(t)$ are such that $u_{m}^{2}, w_{s}^{2}$ have units of power, and $u_{m}^{2} d t, w_{s}^{2} d t$ represent energy packets. Thus, if a "null packet strategy" is adopted, no additional energy is injected in the system and passivity is preserved. Also, even if there is no loss of packets, an empty sampling instance can appear due to increasing delay; in this case the same strategy must be adopted. The drawback of this choice, compared with its alternative (this is, using the value of the previous packet for the new one), is that more energy is eliminated and consequently performance can be degraded. In order to overcome this, the setup pictured at Fig. 7 can be applied. The main idea is to send through the network, at every sampling instance, the sum of all the samples produced up to that time, along with the time at which the information was sent. Sending the time means that, when a packet is received, it can be detected whether it is arriving in the correct order or not. Sending the sum of all samples instead of every sample alone allows for data recovery in case of loss of packets. When a new packet arrives, the subtractor subtracts the values of the previous packet from it, obtaining the number of samples corresponding to the newly arrived data, and a quantity related to the energy contained in them. Then the interpolator distributes the new energy among the number of new samples, pro-

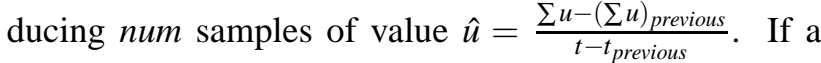
packet is lost or arrives late, the interpolator will produce a "null packet" instance, but the data will be recovered as soon as the next one arrives. The interpolator creates new samples guaranteeing that their energy is not higher than that of the original samples, which preserves passivity. Finally, the samples are placed in a Receive Buffer that provides samples at a constant rate and includes two additional elements, the Compressor and the Expander, that prevent it from overflowing or running empty.

\section{CONTROLLERS}

\subsection{Control Architecture and Strategy}

This system enables a human operator to manipulate a gantry crane placed in a remote location. The operator must be capable of specifying the desired maneuver to be performed by the crane, thus determining the evolution of the position of the crane's load. With this aim a correspondence must be established between the load of the crane and the stylus of the haptic device held by the user. In a proper operation, the sway angle must be minimized, this is, the operator is not allowed to ask for an oscillation of the load. Therefore, in the desired configuration the payload is always placed in the vertical of the trolley from which it is hanging. As a consequence, the desired positions of the girder and trolley are univocally defined by the desired position of the load: its $x$ coordinate corresponds to the displacement of the girder over the rails, its $y$ to the displacement of the trolley over the girder, and its $z$ to the cable length $\rho$. Commands corresponding to these $(x, y, z)$ coordinates are specified with the $(x, y, z)$ position of the stylus.

It must be decided whether to use position control or rate control, this is, whether a displacement of the stylus in the haptic device workspace corresponds to a position or velocity command. If position control is used, an exact equivalence between the two workspaces must be created. This is theoretically possible because the crane workspace is finite, so each one of its points can be assigned to a point in the device workspace. However, since the workspace of the haptic device is much smaller than the workspace of the crane, only a low precision can be achieved. In order to avoid this, we could redefine the origin of joystick coordinates (indexing), but this can distract the user, specially when large time delays are present. Rate control or velocity control, on the other hand, assigns a velocity command to the displacement of the stylus with respect to its zero position, in the same way a classical joystick does. It is not less intuitive for the user than the position control approach, and provides higher precision. Kim et al [13] compared position and rate control for telemanipulators, concluding that the former was more suited for small-workspace tasks, and the latter for wide-workspace tasks, specially for slow ones. Thus, in this application the $x, y, z$ positions of the stylus are used to indicate the desired velocities for the girder $(x)$, trolley $(y)$, and cable length $(\rho)$, while the orientation coordinates are not used.

From the above said, the following controllers (shown 
on Fig. 3) are implemented, and will be described in the rest of this section:

- Two symmetrical impedance controllers (IC) at each side of the scattering transformation, which are taken from the classic bilateral teleoperation architecture proposed in [16]. For a more general introduction to impedance control, see [10]; for a recent application see [21].

- An additional controller block in the wave-variable area, which is labeled "Contr. Pos. Correct." on Fig. 3. It consists of two controllers: one for maintaining passivity of the communications channel with UDP protocol [5], and another one that profits from the energy dissipated in the former for correcting the position drift [8]. For the sake of brevity, these two controllers will not be further described in this paper, and the interested reader is referred to the references for further information.

- An IDA-PBC controller at the crane side. It shapes the potential energy of the plant and injects an appropriate damping, thus suppressing the oscillations of the payload, which is a fundamental issue in operation of cranes.

\subsection{IDA-PBC plant controller}

A 3DoF gantry crane is a mechanical system whose behavior can be described using the port-Hamiltonian formalism [25] as

$$
\left\{\begin{aligned}
\dot{x} & =[J(x)-R(x)] \frac{\partial H}{\partial x}+G(x) u \\
y & =G^{T}(x) \frac{\partial H}{\partial x}
\end{aligned}\right.
$$

where $x=(q, p)^{T}$ are the hamiltonian state coordinates (positions and moments), $u$ the system inputs, $H$ the Hamiltonian (energy) function. Moreover $J(x)=$ $-J^{T}(x)$ and $R(x)=R^{T}(x) \succeq 0$ are respectively the interconnection and the damping matrices. It is assumed that:

- The payload is represented by a point mass $M_{b}$, moving in the $Z$ direction under the actuating force $F_{\rho}$.

- The payload mass and the crane cart are connected by a mass-less, rigid rope of length $\rho$.

- $\theta$ is the swing angle of the payload in an arbitrary direction $\phi$.

- The cart, with mass $M_{c}$, moves on the girder (with mass $M_{r}$ ) in the $Y$ direction under the actuating force $F_{y}$ and both girder and cart move in the $X$ direction under the actuating force $F_{x}$. The cart's position is given by $c=(x, y, 0)$.

Adopting polar coordinates for the payload, the ball coordinates $b$ with $\theta$ as the swing angle result in $b=$ $c-\rho \hat{v}$ with $\hat{v}=(-\sin \theta \sin \phi,-\sin \theta \cos \phi, \cos \theta)^{T}$. The kinetic energy is given by

$$
K=\frac{1}{2}\left(M_{r} \dot{x}^{2}+M_{c}<\dot{c}, \dot{c}>+M_{b}<\dot{b}, \dot{b}>\right)
$$

where $\langle *, *>$ means scalar product, and the potential energy

$$
V=-M_{b} g \rho \cos \theta
$$

where $g$ is the gravity acceleration. The Hamiltonian is $H(q, p)=\frac{1}{2} p^{T} M^{-1}(q) p+V(q)$, where

$$
M(q)=\left[\begin{array}{ccc}
M_{b}+M_{c}+M_{r} & 0 & \ldots \\
0 & M_{b}+M_{c} & \ldots \\
M_{b} s \phi s \theta & M_{b} c \phi s \theta & \ldots \\
M_{b} \rho s \phi c \theta & M_{b} \rho c \phi c \theta & \ldots \\
M_{b} \rho c \phi s \theta & -M_{b} \rho s \phi s \theta & \ldots \\
M_{b} s \phi s \theta & M_{b} \rho s \phi c \theta & M_{b} \rho c \phi s \theta \\
M_{b} c \phi s \theta & M_{b} \rho c \phi c \theta & -M_{b} \rho s \phi s \theta \\
M_{b} & 0 & 0 \\
0 & M_{b} \rho^{2} & 0 \\
0 & 0 & M_{b} \rho^{2} s^{2} \theta
\end{array}\right]
$$

is the mass matrix, with the notation $c \phi=\cos \phi, c \theta=$ $\cos \theta, s \phi=\sin \phi, s \theta=\sin \theta$. The movement equations can be easily derived, obtaining (4) with

$J=\left[\begin{array}{cc}0 & I_{3 \times 3} \\ -I_{3 \times 3} & 0\end{array}\right], R=\left[\begin{array}{cc}0 & 0 \\ 0 & D(x)\end{array}\right], G=\left[\begin{array}{c}0 \\ G_{u}\end{array}\right]$

where $D(x)=0_{5 \times 5}$ if no dissipation is assumed, and $G_{u}=\left[I_{3 \times 3} ; 0_{2 \times 3}\right]$. For a desired configuration in the state space, it is possible to find a state feedback law $u=\beta(x)$ such that the dynamics of the resulting closed loop system is given by $\dot{x}=\left[J_{d}(x)-R_{d}(x)\right] \frac{\partial H_{d}}{\partial x}$, where $J_{d}(x)$ and $R_{d}(x) \succ 0$ are desired interconnection and damping matrices respectively. This system has an energy function $H_{d}(q, p)$ that can be represented as

$$
H_{d}(q, p)=\frac{1}{2} p^{T} M_{d}^{-1}(q) p+V_{d}(q)
$$

The system can be regulated to $x_{d}$ in a passive way if the desired energy function $H_{d}(x)$ assumes a minimum on the state space. This procedure is called Interconnection and Damping Assignment (IDA), and can be applied jointly [18] with Passivity Based Control (PBC). In PBC the control input is naturally decomposed into two terms,

$$
u=u_{d i}(q, p)+u_{e s}(q, p)
$$

where

$$
u_{d i}(q, p)=-K_{v} G_{u}^{T} \frac{\partial H_{d}}{\partial p}
$$


with $K_{v} \succ 0$ responsible for damping injection. Energy shaping is obtained with

$$
u_{e s}=\left(G_{u}^{T} G_{u}\right)^{-1} G_{u}^{T}\left(\frac{\partial V}{\partial q}-\frac{\partial V_{d}}{\partial q}\right)
$$

as in [19]. The desired closed loop dynamics is chosen such that $M_{d}(q, p)=M(q, p)$ and

$$
\begin{aligned}
V_{d}(q)= & \frac{1}{2}\left[\left(k_{x}\left(x-x_{d}\right)^{2}+k_{y}\left(y-y_{d}\right)^{2}\right)\left(1+\rho_{d}^{2} k_{\theta} \sin \theta\right)\right. \\
& \left.+k_{\rho}\left(\rho-\rho_{d}\right)^{2}\right]+M_{b} g(1-\cos \theta) \rho
\end{aligned}
$$

where $x_{d}, y_{d}, \rho_{d}$ with $\theta=0$ defines the desired equilibrium point. It should be noted that the purpose of including the term $\left(1+\rho_{d}^{2} k_{\theta} \sin \theta\right)$ is to help injecting dissipation in the non-actuated coordinate, $\theta$. Since the effects of the oscillations can be projected into the actuated $(x, y)$ axes, the corresponding control actions can be used to minimize them. Similar ideas can be applied to other underactuated systems, thus generalizing this result. For this particular case, the resulting control actions are

$$
u=\underbrace{\left[\begin{array}{c}
k_{x}\left(1+\rho_{d}^{2} k_{\theta} \sin \theta\right)\left(x_{d}-x\right) \\
k_{y}\left(1+\rho_{d}^{2} k_{\theta} \sin \theta\right)\left(y_{d}-y\right) \\
k_{\rho}\left(\rho_{d}-\rho\right)-M_{b} g
\end{array}\right]}_{u_{e s}}-\underbrace{\left[\begin{array}{c}
k_{v x} \dot{x} \\
k_{v y} \dot{y} \\
k_{v \rho} \dot{\rho}
\end{array}\right]}_{u_{d i}}
$$

\subsection{Impedance controllers}

Impedance matching may be used to avoid wave reflections, which can be achieved if both sites are placed under velocity control [16]. This entails a configuration such as in Fig. 8, where two symmetric controllers are placed at each end of the transmission channel. Thus, each side is receiving force information and providing velocity signals. In Fig. $8, \dot{x}_{c r}=(\dot{x}, \dot{y}, \dot{\rho})^{T}$, and, since the dynamics of the haptic device is negligible, $v_{h}=\dot{x}_{m}, f_{h}=f_{m}$. Notice also that the spring-damper at the right-hand side of the figure performs damping injection and part of the IDA-PBC energy shaping, by the assignments $B_{s}=K_{v}, K_{s}=\left(k_{x}, k_{y}, k_{\rho}\right)^{T}, v_{s}=\left(\dot{x}_{d}, \dot{y}_{d}, \dot{\rho}_{d}\right)^{T}$.

The equations of the controllers can be written as:

$$
\begin{gathered}
f_{m}=K_{m} \int\left(\dot{x}_{m}-v_{m}\right) d t+B_{m}\left(\dot{x}_{m}-v_{m}\right) \\
f_{s}=K_{s} \int\left(v_{s}-\dot{x}_{c r}\right) d t+B_{s}\left(v_{s}-\dot{x}_{c r}\right)
\end{gathered}
$$

In this way, we are not only transmitting energy through the strings $K_{m}, K_{s}$, but also dissipating some of it in the dampers $B_{m}, B_{s}$. Consequently, artificial damping is injected, which is a requisite to ensure a passive behavior when, as in this case, a mechanical system is interacting with the environment. The parameters of this controller are the stiffness constants of the springs, $K_{m}$ and $K_{s}$, and the viscous frictions, $B_{m}$ and $B_{s}$. If the latter are chosen to be equal to the scattering parameter of the transmission line, $B$, no wave reflections will take place [16].

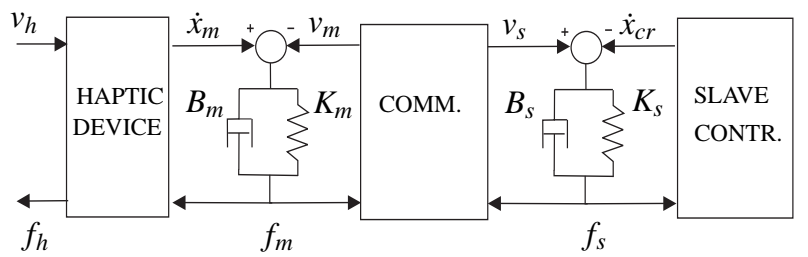

Fig 8: Symmetric Impedance Controllers

\section{HUMAN-MACHINE INTERFACE}

The Human-Machine Interface (HMI) [9] consists of:

- A graphical interface which provides the operator with visual information of the crane's workspace.

- A haptic interface where force is fed back to the operator. This consists of the "original" force originated from the teleoperation setup, augmented by a new haptic feedback.

\subsection{Force Feedback}

In a bilateral teleoperation scheme, the feedback force reflects the force moving the slave device, as well as the environmental contact force. By feeling the forces that are entering the crane as control inputs $\left(F_{\text {motors }}\right)$, the operator can intuitively perceive the effort that he is demanding from the actuators: in the $x$ axis, the force needed to move the girder along the rails; in the $y$, to move the trolley along the girder; and in the $z$ axis, to lift or lower the payload. Consequently he feels, at a certain level, that he is moving the crane himself, thus getting a sense of telepresence. This is what could be called the "classical" force feedback.

Additionally, other forces which help the operator to command the system in a safe way can be programmed. These are repulsion forces near the workspace limits and known obstacles. These forces make the operator intuitively avoid those areas where operation is dangerous. In the gantry crane case, the workspace is clearly limited, so it is possible to program those repulsion forces in the virtual environment, acting on the position setpoint introduced by the operator. Additionally, if the obstacles present in the workspace are known, a repulsion force with respect to those obstacles can also be programmed. Thus, if the operator tries to carry the load to a zone close to (a) the limits of the workspace, or (b) an obstacle, he feels an opposition that makes him decrease the speed, increasing the safety. The shape of these forces is

$$
F_{\text {repulsion }}=\left\{\begin{aligned}
K_{r} d+B_{r} \dot{d} & \text { if }|d|<|D| \\
0 & \text { if }|d| \geq|D|
\end{aligned}\right.
$$

where $d$ is the distance from the position setpoint to the virtual obstacle, $D$ is the minimum distance required for the activation of the repulsion force, and $K_{r}, B_{r}$ are 
constants corresponding to a spring and a damper, respectively. These are contact forces with a virtual environment, and are exerted based on the position setpoint specified by the operator, which is a virtual position and not the real position of the payload. The overall force is

$$
F_{\text {overall }}=F_{\text {motors }}+F_{\text {repulsion }}
$$

If no contact with the environment is taking place, the operator shall feel a small feedback force $\left(F_{\text {motors }}\right)$, that helps him adequate his commands to the dynamics of the system, but that does not unnecessarily disturb or distract him. Generally speaking, when the crane is moving in free space the operator must feel that the movement is mainly free, even when he perceives some force (due to the presence of frictions, even maintaining a constant velocity requires some effort). $F_{\text {repulsion }}$ is felt as qualitatively different, and it acts as a warning of a dangerous maneuver.

\subsection{Visual Feedback}

The need of visual feedback for the operator implies sending information from the slave side to the master side, and transforming it into images that are refreshed in real time. There are several ways to achieve this:

- Option A: Sending video signal from a webcam placed on the remote plant and connected to the slave computer. This has two disadvantages: in order to have real time vision, a low refresh time is needed, which requires transmitting a large amount of information at each sampling time and therefore a large bandwidth. Besides, due to limitations of the camera or the software, the image may not have enough quality to be correctly interpreted by the operator.

- Option B: It may be possible to create a virtual reality model of the remote environment at the master computer and to render it to the operator. During operation, information about the crane position is being received at the master computer and the virtual environment is modified accordingly, producing an animation that reflects the evolution of the plant. With this option no additional data must be sent, thus leaving the communications channel unmodified. Besides, the generated image has a simplicity that facilitates the operator's task. In our case, the software used for controlling the master side (a Simulink environment) is already suitable for this modeling, as it includes a Virtual Reality toolbox. A drawback of this option is that an a priori knowledge of the elements present at the workspace (platforms or obstacles) is needed, as they must be modeled in order to be taken into consideration by the operator. Any modification of the workspace results in a new environment which must be modeled again.

- Option C: Using both a virtual environment on the master side and a webcam on the slave side, but this time connected to an artificial vision system. This system reads the images acquired by the camera and extracts its fundamental properties. Only the necessary data (size and position of the objects that appear in the workspace) are sent to the master side. The master computer reconstructs the virtual environment according to these selected data. Thus, we obtain:

- Information about unpredicted modifications of the workspace.

- The required bandwidth is smaller than that needed for sending video images.

- The operator sees clear, virtually generated images, instead of low quality video images.

We have implemented option B, designing an operator screen that displays the virtual environment, see Fig. 9. The image corresponds to the workspace configuration that was used in the experiments, with a brown obstacle at the center and two platforms at each side. The real (measured) position of the payload is represented by a small pink box, while the position setpoint is pictured in blue.

\section{EXPERIMENTS}

We show here the result of a teleoperation maneuver, whose goal is to carry the payload from an original platform to a final one, avoiding an obstacle placed between them. Both platforms and the obstacle were modeled as blocks and included at the virtual environment. Visually, they resemble the real elements, although with some degree of transparency so that the operator can see through them, thus having a more detailed view of the scene. The workspace limits and the obstacle were modeled as virtual walls, so the operator is not able to produce any position set-point that would imply passing through them. Additionally, they produce repulsion forces as explained in section 5.1., so that the user adapts his commands to them and tends to avoid any collision. A picture of the maneuver is shown in Fig. 10.

In this experiment the data was sent via internet, with the transport protocol UDP. In practice, both master and slave were physically placed at the same laboratory, but a software application was developed [6] and implemented in order to emulate in real time the characteristics of actual Internet communication. This was carried out in the following way: firstly, a remote location was chosen in a distant place, namely Japan. It was observed that communication delays to this location ranged from 150 to $300 \mathrm{msec}$ and that there was a $1 \%$ loss of data 


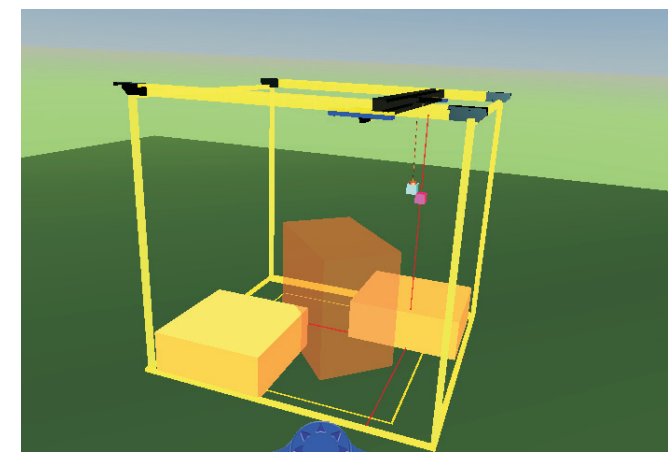

Fig 9: Screenshot of the visual interface

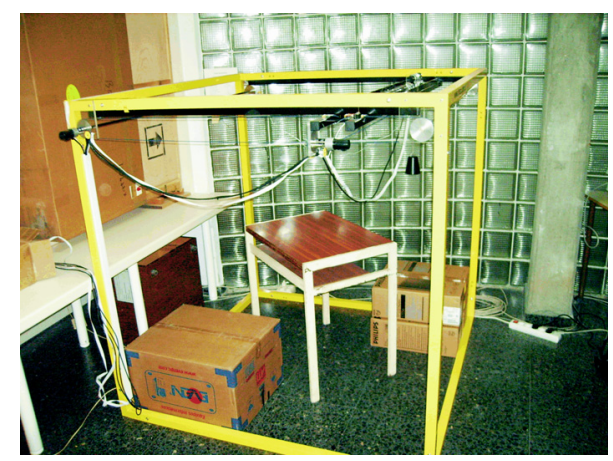

Fig 10: Maneuver

packets. During teleoperation, a "relay demon" measures in real time the delays and losses of packets towards this remote location. Simultaneously, it reproduces them on each of the data packets that come to it towards/from the controller/slave. In this way, the behavior of the net is exactly reproduced, and the resulting system is equivalent to having an operator in Spain and the crane in Japan.

In order to evaluate the efficiency of the interface, an operator was asked to perform the maneuver with and without the haptic features. In the latter case, the teleoperation setup proposed in section 2 was used, as well as the visual interface presented in section 5.2., but the force feedback was not augmented in the way suggested in section 5.1.. In the former case the operator could benefit from all the features.

The operator reported that the proposed haptic features were useful in the following way: with them, he could intuitively perceive when he was going to enter a dangerous zone, without needing to pay excessively detailed attention to the visual feedback he was receiving. Hence, he felt more confident and was able to concentrate on the task of commanding the crane.

Without repulsion forces, on the other hand, the user had to move slowly around the points where visibility was not perfectly clear, as a faster maneuver could result in a collision. This resulted in a more hesitating command, and in longer (about 20\%) completion times.

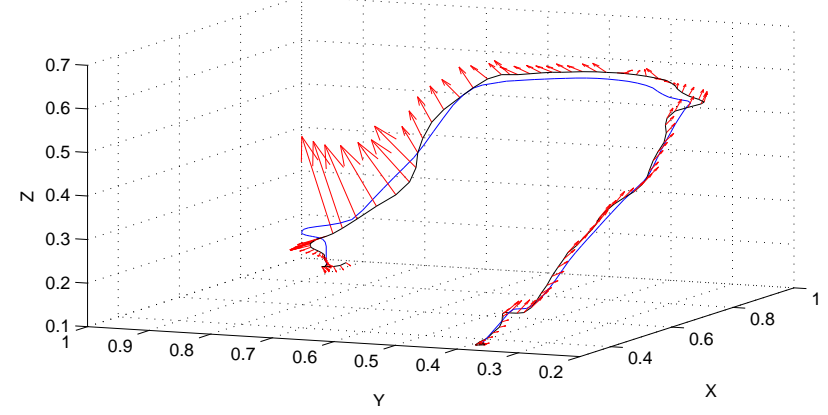

Fig 11: Operation with haptic interface

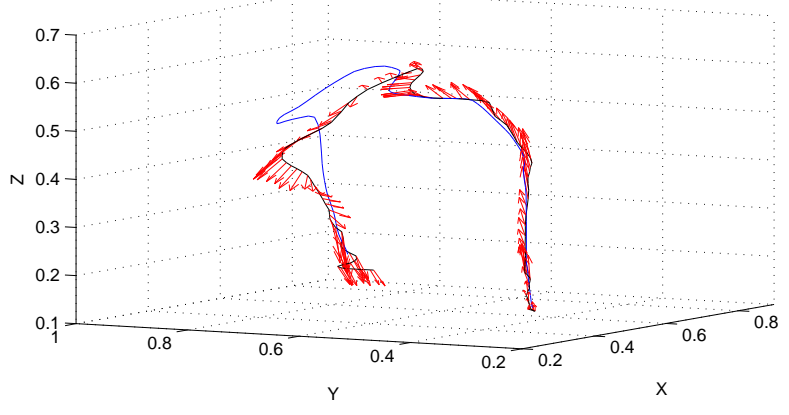

Fig 12: Operation without haptic interface

Two representative results of these maneuvers are shown in Fig. 11 for the former case (with haptic features), and in Fig. 12 for the latter. The position setpoints are represented as a blue line, the trajectory of the payload as a black line, and the red arrows are the forces felt by the operator. It can be noticed that in the first picture a large force warns the user that he has entered the surroundings of the obstacle, so he must lift the payload higher. This effect is not felt in the second case, so the user must rely more heavily on visual feedback. In this particular case, the augmented maneuver was completed in 30 seconds while the non-augmented one took 35 seconds. These were typical results.

\section{CONCLUSIONS AND FUTURE WORK}

A configuration for remotely operating an electromechanical system through the internet has been presented, and applied to the case study of a gantry crane. Passivity methods have been used at every part of the teleoperation setup (master, communication channel, and slave) in order to ensure the stability of the whole system. The main issues in teleoperated control (stability in presence of time delays) and in control of underactuated systems, specifically cranes (suppression of undesired oscillations), have been conveniently addressed. A digital, unreliable internet-based communication channel has been considered in theory and practice. 
A key feature is that a virtual environment, including visual and haptic feedback, has been added in order to enhance the setup. It has been shown that the use of this environment in combination with passivity-based control techniques makes it possible to remotely operate a crane in a safe, robust way. This has been validated with experiments where the use of the haptic interface improved performance even with large, variable time delays.

We believe that the methodology and solutions adopted here have enough generality to be extended to other systems. Thus, the same scheme can be applied for teleoperating other mechanical devices, by adapting the virtual environment and the IDA-PBC controller.

Further improvements may be focused on enhancing the visual feedback to the operator, as mentioned in section 5.2. (option C). Additionally, operator tests should be conducted on a larger scale, in order to obtain statistical results about the performance improvements of the interface.

\section{REFERENCES}

[1] E.M. Abdel-Rahman, A.H. Nayfeh, and Z.N. Masoud, "Dynamics and control of cranes: A review," Journal of Vibration and Control, vol. 9, no.7, pp. 863 - 908, 2003.

[2] M.A. Al-Mouhamed, M. Nazeeruddin, and S.M.S. Islam, "Experimental evaluation of feedback modalities for five teleoperation tasks," IEEE Trans. on Instrumentation and Measurement, vol. 59, pp. 361 - 371, 2010.

[3] R.J. Anderson and M.W. Spong, "Bilateral control of teleoperators with time delay," IEEE Trans. on Automatic Control, vol. 5, pp. 494 - 501, 1989.

[4] D. Blackburn, W. Singhose, J. Kitchen, V. Patrangenaru, J. Lawrence, T. Kamoi, and A. Taura, "Command shaping for nonlinear crane dynamics," Journal of Vibration and Control, vol. 16, no.4, pp. 477 - 501, April 2010.

[5] N. Chopra, P. Berestesky, and M.W. Spong, "Bilateral teleoperation over unreliable communication networks," IEEE Trans. on Control Systems Technology, vol. 16, no. 2, pp. 304 - 313, 2008.

[6] M. Díaz-Cacho, A. Fernández Villaverde, M. García, and A. Barreiro, "Internet emulation system for udp teleoperation," Proc. of the 16th IEEE Med. Conf. on Control and Automation, pp. 1417 - 1422, 2008.

[7] A. Fernández Villaverde, A. Barreiro, and C. Raimúndez, "Digital passive teleoperation of a gantry crane," Proc. of the IEEE Int. Sympos. Industrial Electronics, pp. 56 - 61, June 2007.

[8] A. Fernández Villaverde, A. Barreiro, and C. Raimúndez, "Passive position error correction in internet-based teleoperation," Automatica, vol. 46, pp. 1884 - 1890, 2010.

[9] A. Fernández Villaverde, M. Díaz-Cacho, A. Barreiro, and C. Raimúndez, "Haptic interface for crane teleoperation," Proc. of the 8th Portuguese Conf. Automatic Control, pp. 46 - 51, 2008.

[10] N. Hogan, "Impedance control - an approach to manipulation," Trans. of the ASME UU Journal of Dynamic Systems, Measurement, and Control, vol. 107, pp. 1 - 24, March 1985.

[11] P.F. Hokayem and M.W. Spong, "Bilateral teleoperation: An historical survey," Automatica, vol. 42, pp. 2035 - 2057, 2006.

[12] C-S Kim and K-S Hong, "Boundary control of container cranes from the perspective of controlling an axially moving string system," International Journal of Control, Automation and Systems, vol. 7, pp. 437 - 445, 2009.

[13] W. S. Kim, F. Tendick, S. Ellis, and L. Stark, "A comparison of position and rate control for telemanipulations with consideration of manipulator system dynamics," IEEE Journal of Robotics and Automation, vol. 3, pp. 426 - 436, 1987.

[14] K.A.F. Moustafa, M.B. Trabia, and M.I.S. Ismail, "Modelling and control of an overhead crane with a variable length flexible cable," International Journal of Computer Applications in Technology, vol. 34, pp. 216 - 228, 2009.

[15] S. Munir and W. J. Book, "Internet-based teleoperation using wave variables with prediction," IEEE/ASME Trans. on Mechatronics, vol. 7, no. 2, pp. 124 - 133, June 2002.

[16] G. Niemeyer and J-J.E. Slotine, "Stable adaptive teleoperation," IEEE Journal of Oceanic Engineering, vol. 16, no. 1, pp. 152 - 162, January 1991.

[17] E. Nuño, L. Basáñez, and R. Ortega, "Passivitybased control for bilateral teleoperation: A tutorial," Automatica, vol. 47, pp. 485 - 495, 2011.

[18] R. Ortega, A.J. van der Schaft, B. M. Maschke, and G. Escobar, "Interconnection and damping assignment passivity-based control of port-controlled hamiltonian systems," Automatica, vol. 38, no. 4, pp. 585 - 596, April 2002.

[19] R. Ortega, M. W. Spong, F. Gómez-Estern, and G. Blankenstein, "Stabilization of a class of underactuated mechanical systems via interconnection and damping assignment," IEEE Trans. on Automatic Control, vol. 47, no. 8, pp. 1218 - 1233, 2002.

[20] M. Rossi, K. Tuer, and D. Wang, "A new design paradigm for the rapid development of haptic and telehaptic applications," Proc. of the IEEE Conf. Control Applications, pp. 1246 - 1250, 2005.

[21] M. Salehi and G. Vossoughi, "Impedance control of flexible base mobile manipulator using singular perturbation method and sliding mode control law," 
International Journal of Control, Automation, and Systems, vol. 6, pp. 677 - 688, 2008.

[22] J.K. Salisbury, F. Conti, and F. Barbagli, "Haptic rendering: introductory concepts," IEEE Computer Graphics and Applications, vol. 24, no. 2, pp. 24 32, March/April 2004.

[23] S. Stramigioli, C. Secchi, A.J. van der Schaft, and C. Fantuzzi, "Sampled data systems passivity and discrete port-hamiltonian systems," IEEE Trans. on Robotics, vol. 21, pp. 574 - 587, 2005.

[24] J.J. Troy, C.A. Erignac, and P. Murray, "Haptics enabled UAV teleoperation using motion capture systems," Journal of Computing and Information Science in Engineering, vol. 9, pp. 011003, 2009.

[25] A.J. Van der Schaft and B. Maschke, "The hamiltonian formulation of energy conserving physical systems with external ports," Archiv für Elektronik und Übertragungstechnik, vol. 49, pp. 362 - 371, 1995.

[26] J. Vaughan, D. Kim, and W. Singhose, "Control of tower cranes with double-pendulum payload dynamics," IEEE Trans. on Control Systems Technology, vol. 18, pp. 1345 - 1358, 2010.

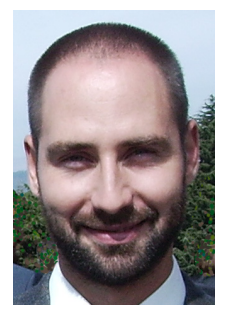

Alejandro F. Villaverde received the MEng and PhD degrees in Electrical Engineering from the University of Vigo in 2004 and 2009, respectively. He is at the Spanish National Research Council (CSIC), where he works on system identification and optimization techniques in systems biology. Previous research interests include teleoperation, passivitybased control, reset control, and haptics. He has been a visiting researcher at Stanford, MIT, and the University of Groningen.

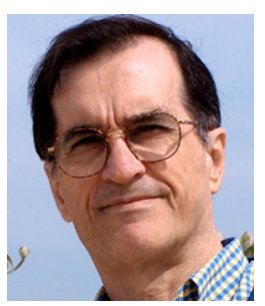

Cesáreo Raimúndez received the Electronic Engineering degree from the University of São Paulo in 1969, and the $\mathrm{PhD}$ from the University of Vigo in 1996. Currently he is an Associate Professor in the Departament of Systems Engineering and Control at the University of Vigo. His research focuses on control of underactuated nonlinear systems using passivity and on adaptive control.

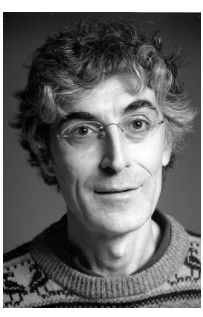

Antonio Barreiro received the Industrial Engineering and $\mathrm{PhD}$ degrees from the Polytechnic University of Madrid (UPM) in 1984 and 1989, respectively. From 1984 to 1987 he was with the Department of Applied Mathematics at the UPM. Since 1987 he has been with the Departament of Systems Engineering and Control at the University of Vigo, where he is now Professor in the area of Automatic Control. His research interests include nonlinear and robust stability, time-delay systems and reset control, with applications in networked control and teleoperation systems. 